\title{
Effective stress in rock
}

\author{
I Gray Sigra Pty Ltd, Australia
}

\begin{abstract}
This paper looks at what is the meaning of effective stress in rock and how it is measured. The paper examines the field measurement of stress in intact and jointed systems including fluid pressure and how it acts within the matrix and joints. How the fluid pressure acts varies between rock types and structure. The processes of stress measurement by overcoring and hydrofracture are considered and the benefits and deficiencies of each system discussed. The determination of Biot's poroelastic coefficient and how this relates to a fractured system are discussed. The importance of effective stress on failures within rock are considered.
\end{abstract}

Keywords: stress, fluid, pressure, rock, effective, poroelastic, joints, Biot

\section{Introduction}

The nature of effective stress in rock is generally ignored with analysis being conducted on the basis of total stress or in making the assumption that fluid pressure acts throughout the rock mass as in a soil. These assumptions can lead to serious errors in stress measurement and the models on which rock engineering is based. Appropriately implemented effective stress models in rock mechanics will greatly enhance our understanding of rock mass behaviour as it has done for soil mechanics. However the effective stress equation is not as simple in a rock as in a soil.

The differences between rock and soil are important. Rock has fabric at grain or crystalline scale, it almost always has joints of varying size and orientation and it usually has some form of porosity. How fluid pressure acts at an intergranular level, within the porosity or within joints is important to the stability of a rock mass. Rock failure may be thought to be due to the extension of fractures that then coalesce to form a failure plane.

The area and manner in which fluid acts upon this failure surface changes from that acting in the rock mass or the joint infill to one in which it acts upon the surfaces of the sliding joint. These are likely to be very different and to change through the failure process. If the rock is sedimentary then the fluid pressure acting within pore space may be very important. If however the rock is obsidian then the pore space is negligible, it lacks crystalline structure and the only place fluid pressure can act is within fractures. Whether the fractures are open or filled, and what they are filled with, becomes very important as it affects how the fluid may act within them.

Practically most rocks will have porosity and jointing. To be able to analyse their behaviour properly it is essential to know the magnitude (pressure and fractional ratio) and orientation in which fluid pressure acts.

\section{The definition of effective stress}

Effective stress in the ground is the total stress minus some fraction of fluid pressure. It can be expressed mathematically in Equation (1).

$$
\sigma_{i j}^{\prime}=\sigma_{i j}-\delta_{i j} \alpha_{i} P
$$

where:

$\sigma_{i j}^{\prime} \quad$ is the effective stress on a plane perpendicular to the vector $i$ in the direction $j$.

$\sigma_{i j} \quad$ is the total stress on a plane perpendicular to the vector $i$ in the direction $j$.

$\delta_{i j} \quad$ is the Kronecker delta. If $i \neq j$ then $\delta_{i j}=0$, while if $i=j$ then $\delta_{i j}=1$. 
$\alpha_{i} \quad$ is Biot's coefficient affecting the plane perpendicular to the vector $i$. It lies between 0 and 1 .

$P \quad$ is the fluid pressure in pores and fractures within the rock.

The Kronecker delta term is used because a static fluid cannot transmit shear.

The directional subscript indicating direction in Biot's coefficient is not usual practice where, for measurement reasons, only a scalar value is obtained.

Equation (1) is important. It states that the effective stress in rock is generally greater than would be the case for a loose grained sand with point contact between particles. In the case of a loose grained sand Biot's coefficient can be readily understood and experimentally shown to be unity. A Biot's coefficient of unity can also be applied to finer soils such as clays (Terzaghi \& Peck 1948).

In Equation (1), $\alpha_{i}$ represents the fraction of the area of the plane perpendicular to the vector $i$ over which the fluid pressure acts. However Biot's coefficient (Biot \& Wills 1957) is a function of poroelastic behaviour. This means that its effect is really dependent on the stiffness of the rock matrix. This can be easily explained mathematically. The general equation for deformation of a porous rock or soil with a fluid pressure may be expressed in Equation (2).

$$
\varepsilon_{i i}=\frac{1}{E_{i}} \sigma^{\prime}{ }_{i i}-\frac{v_{j i}}{E_{j}} \sigma_{j j}^{\prime}-\frac{v_{k i}}{E_{k}} \sigma_{k k}^{\prime}
$$

where:

$$
\begin{aligned}
& \varepsilon_{i i} \quad \text { is the strain in the } i \text { th direction under the influence of the three principal effective stresses. } \\
& E_{i} \quad \text { is the Young's modulus in the } i \text { th direction. } \\
& v_{i j} \quad \begin{array}{l}
\text { is Poisson's ratio describing the negative ratio of deformation in the } j \text { th direction to } i \text { th } \\
\text { direction under a direct stress in the } i \text { th direction. }
\end{array} \\
& \begin{array}{ll}
\sigma_{i i}^{\prime} & \text { is the effective stress in the } i \text { th direction. } \\
i & =1,2,3 . \\
j & =2,3,1 . \\
k \quad=3,1,2 .
\end{array}
\end{aligned}
$$

The subscripts $i, j, k$ indicate the directions of the three principal effective stresses.

Substituting Equation (1) for effective stress into Equation (2) and using changes in stress, pressure and strain it is possible to write Equation (3).

$$
\Delta \varepsilon_{i i}=\frac{1}{E_{i}} \Delta \sigma_{i i}-\frac{v_{j i}}{E_{j}} \Delta \sigma_{j j}-\frac{v_{k i}}{E_{k}} \Delta \sigma_{k k}-\Delta P\left(\frac{1}{E_{i}} \alpha_{i}-\frac{v_{j i}}{E_{j}} \alpha_{j}-\frac{v_{k i}}{E_{k}} \alpha_{k}\right)
$$

Equation (3) shows how changing fluid pressure and Biot's coefficients affect strain in a three-dimensional elastic system.

Normally Biot's coefficient is only described as a scalar value derived from measurements of change of volume (Franquet \& Abass 1999). Theoretically these single values are frequently shown to be related to the compressibility of the matrix and compressibility of the grains themselves via Equation (4) (Franquet \& Abass 1999).

$$
\alpha=1-\frac{c_{m a}}{c_{b}}
$$

where:

$c_{m a}$ is the volumetric compressibility of the matrix.

$c_{b} \quad$ is the volumetric compressibility of the minerals comprising the matrix. 
Equations (1) and (3) really show a different aspect of Biot's coefficient. Equation (1) shows it as a ratio that might be thought of as describing the fraction of area of a surface within the rock over which the fluid pressure acts. Equation (3) shows it being related the elastic compressibility of the rock mass. Equation (1) could be applied to a joint with a fractional area open to the effects of fluid pressure while Equation (3) shows the properties of a porous elastic solid.

\section{The measurement of Biot's coefficient}

Most values of Biot's coefficient in rock come from the petroleum industry. These values are arrived at by testing core placed in a metal jacket, usually made of copper. The compressibility of the matrix is determined in a drained test where the matrix is loaded externally. The resulting change in the matrix volume due to the change in applied pressure is measured. The matrix compressibility is the change in pressure per unit change in volume. The compressibility of the material comprising the rock is arrived at by carrying out an unjacketed test where the test fluid penetrates the specimen and the compressibility measured is that of the mineral itself.

A second method used to measure Biot's coefficient is to measure the fluid volume released from a saturated sample during a drained, jacketed test. It can be shown that Biot's coefficient can be described by Equation (5) (Franquet \& Abass 1999).

$$
\alpha=\frac{\Delta V_{p}}{\Delta V}
$$

where:

$\Delta V_{p} \quad$ is the pore volume expelled.

$\Delta V \quad$ is the total external change in volume of the sample.

The measurement of the small volume changes required in either of these test techniques is not a trivial task.

An alternative procedure used by the author involves the use of a core sample that is fitted with strain gauges and is subject to external loading and internal fluid pressure loading. By re-arranging Equation (3) for three principal axes it is possible to arrive at Equation (6) which applies to an orthotropic material.

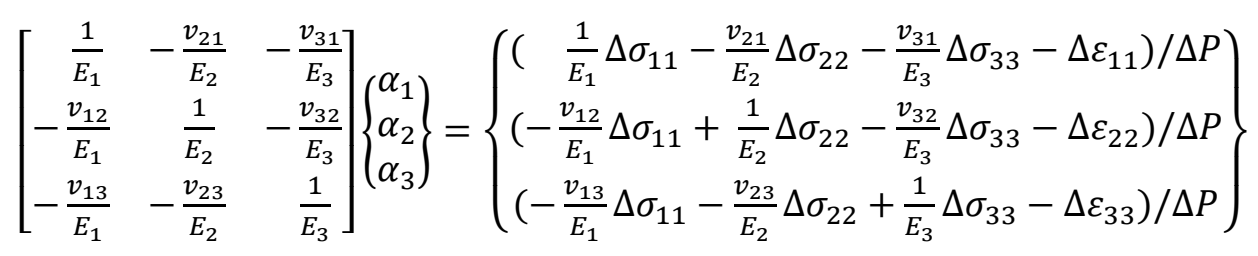

In the case of a cylindrical core being tested with axial and circumferential stress, some assumptions have to be made as the circumferential stress is even and therefore, $\Delta \sigma_{2}=\Delta \sigma_{3}$. Under these conditions it is not possible to determine $v_{23}$ or $v_{32}$ and these have to be estimated. The normal process would be to use an estimate so that these values of Poisson's ratio in the 2-3 plane are equal as are $E_{2}$ and $E_{3}$. This leads to a solution for an axisymmetric sample. It is however possible to deduce a solution for $v_{21}$ from $v_{12}$ as these are related by a form of Betti's (1872) theorem (Daniel \& Ori 2006) in Equation (7).

$$
\frac{v_{i j}}{E_{i}}=\frac{v_{j i}}{E_{j}}
$$

The procedure involves loading the strain gauged core into a triaxial test rig in a stepwise process with progressively increasing axial and circumferential loading so as to avoid failure. This should then be followed by steps of decreasing pressure. The sample can then be re-loaded and have fluid pressure introduced to it and then released whilst measuring the strain change. These steps in fluid pressure can be used in Equation (6) along with the values of Young's modulus and Poisson's ratio existing at the stress level of the test to determine Biot's coefficient in each direction. 


\section{$4 \quad$ Values of Biot's coefficient}

In soils it has been shown that Biot's coefficient is essentially unity because the matrix compressibility is high. Most measurements of Biot's coefficient in rock quote a single value. Testing has focussed on petroleum reservoir rocks which, by their nature, are fairly porous and therefore tend to have a low matrix stiffness compared to the mineral stiffness. Therefore the value of Biot's coefficient could be expected to be high. Values of Biot's coefficient published by Detournay and Cheng (1993) are given in Table 1. Surprisingly these values seem to bear very little relation to porosity.

Table 1 Values of Biot's coefficient from Detournay and Cheng (1993)

\begin{tabular}{llll}
\hline & Porosity (\%) & Permeability (mD) & Biot's coefficient \\
\hline Sandstones & & & \\
\hline Rhur & 2 & 0.2 & 0.65 \\
\hline Berea & 19 & 190 & 0.79 \\
Weber & 6 & 1 & 0.64 \\
\hline Ohio & 19 & 5.60 & 0.74 \\
Pecos & 20 & 20 & 0.83 \\
Boise & 26 & 800 & 0.85 \\
\hline Other rock & & & \\
\hline $\begin{array}{l}\text { Tennessee } \\
\text { marble }\end{array}$ & 0.02 & 0.0001 & 0.19 \\
\hline Charcoal granite & 0.02 & 0.0001 & 0.27 \\
\hline Westerly granite & 0.01 & 0.0004 & 0.47 \\
\hline
\end{tabular}

The values in Table 1 however represent a single value. Franquet and Abass (1999) have shown that Biot's coefficient is variable depending on the state of confinement. An example of this relationship is shown for a particular sample in Figure 1. It shows a reducing value of Biot's coefficient with increasing stress. This behaviour is not a surprise as the modulus of most sedimentary rock changes significantly with stress.

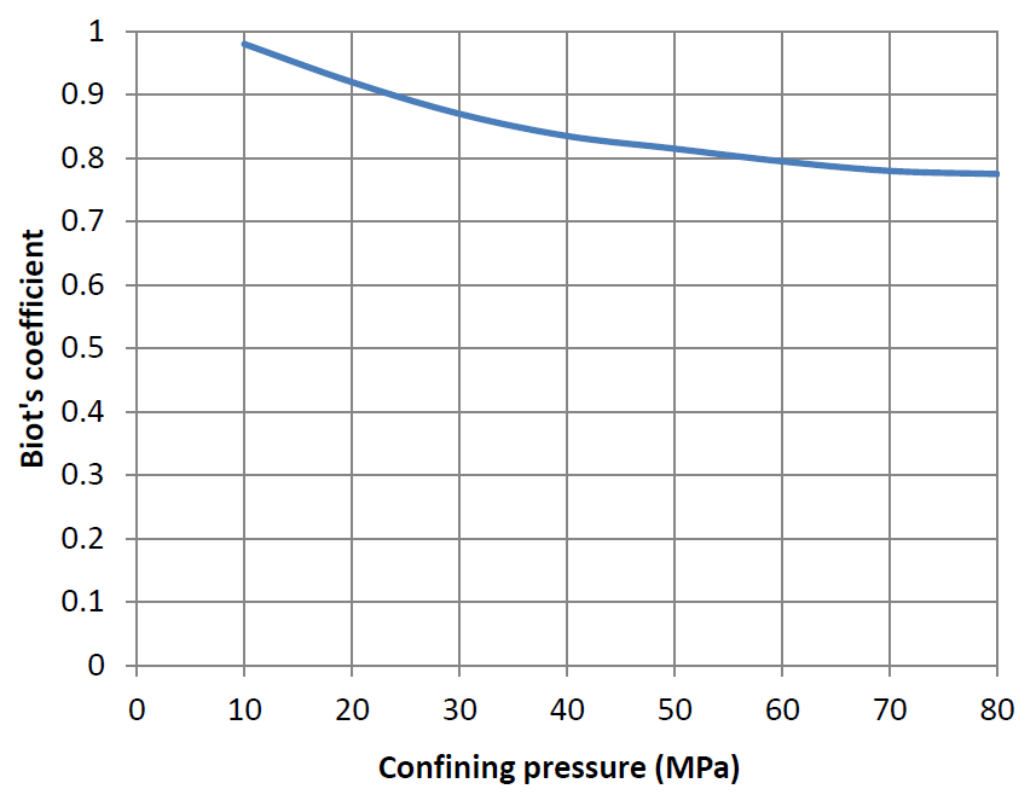

Figure 1 Example of the variation in Biot's coefficient with stress (redrawn from Franquet \& Abass (1999) 
An example of the changing modulus of a sample of Hawkesbury sandstone from Sydney is shown in Figure 2. It has been loaded triaxially in approximately $2.5 \mathrm{MPa}$ steps. The sample was a fairly porous, medium grained, quartzitic sandstone with no visible bedding. It shows a very low stiffness without confinement. This stiffness however increases dramatically with average stress.

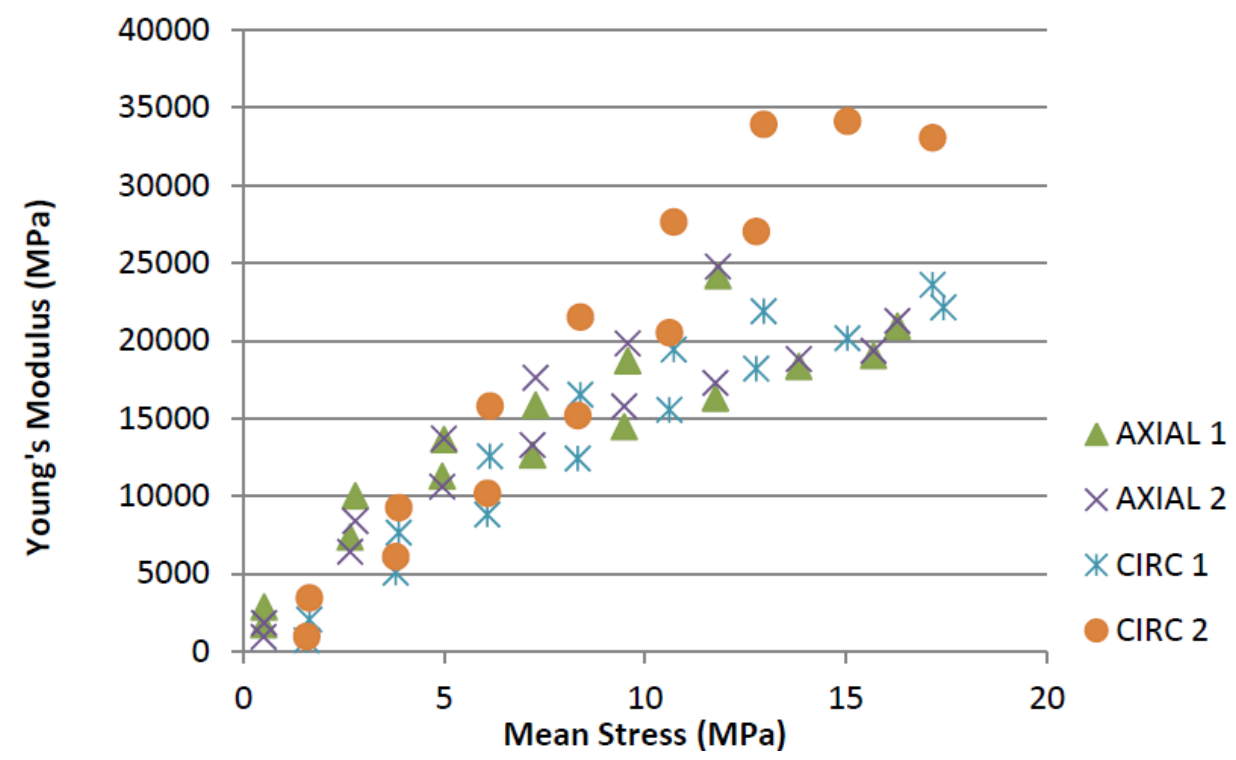

Note: Axial 1 is the vertical stiffness obtained from rosette 1 . Axial 2 is the vertical stiffness obtained from rosette 2 . Circ 1 is the horizontal stiffness obtained from rosette 1 . Circ 2 is the horizontal stiffness obtained from rosette 2.

Figure 2 Variation in Young's modulus with axial stress in a Hawkesbury sandstone sample. The sample has been subject to progressive axial and confining stress

The sample was tested for Biot's coefficient over the stress range and showed a variation of only 0.38 to 0.44 in both vertical and horizontal orientations over the stress range up to $18 \mathrm{MPa}$.

\section{$5 \quad$ Stress measurement and Biot's coefficient}

The two prime means of determining in situ stress are hydrofracture and overcoring.

Hydrofracture is the prime means used by the petroleum industry to measure the minor principal stress at great depth. It is also used by others at shallower depths.

The analysis of hydrofracturing is based upon using fluid pressure in a borehole to generate a tensile stress at a borehole wall which overcomes the tensile rock strength and the tangential stresses at the borehole wall generated by the far field stresses. The hole is then allowed to depressurise by leak off and the closure stress of the generated fracture is determined. This pressure is related to the minimum principal stress. The hole may then be re-opened by re-pressurisation to theoretically determine the opening pressure without the effect of the tensile strength of the rock.

Haimson and Fairhurst (1969) allow for the effects of Biot's coefficient on the opening pressure of a hydraulic fracture. Within the range of Biot's coefficient and Poisson's ratio the difference between the fracture initiation pressure and the pore pressure may be a factor of two. The closure pressure, which is a direct measurement of the minimum total stress, is not apparently dependent on Biot's coefficient. The interpretation of the minimum effective stress is however by definition dependent on Biot's coefficient.

Overcoring has traditionally been undertaken in boreholes that are inclined upwards so as to lead to drainage of the hole and cleanliness so as to permit the gluing of strain gauges to the wall of the pilot hole. The analysis used has been based upon elastic principals of solids that do not exhibit poroelastic behaviour. As overcoring is now being regularly conducted in deep vertical holes, sometimes exceeding $1 \mathrm{~km}$ in depth (Gray 2000; Gray et al. 2013), allowance must be made for pore pressure and how it acts. Equation (8) has been developed to 
describe the change in diameter that occurs in a pilot hole allowing for the effects of fluid pressure in the overcore hole. It both includes an even pore fluid pressure throughout the overcore cylinder and the effects of external fluid pressure on the cylinder.

$$
\left.\Delta D_{i}=\frac{D}{E}\left[2 \sigma_{m}+4 \sigma_{D}\left(1-v^{2}\right) \cos 2 \theta_{i}-v \sigma_{z}-\left((1-2 v)+(1+v) \alpha_{r}\right) p\right)\right]
$$

where:

$D \quad$ is the pilot hole diameter.

$\Delta D_{i}$ is the change in pilot hole diameter at angle $\theta_{i}$ from the principal stress direction.

$E \quad$ is Young's modulus perpendicular to the hole.

$p \quad$ is the fluid pressure at the overcore location.

$\sigma_{m}$ is the mean total stress acting perpendicularly to the axis of the hole.

$\sigma_{D} \quad$ is the deviatoric (major stress - minor stress) $/ 2$ stress acting perpendicularly to the hole.

$\sigma_{z} \quad$ is the total stress acting on a plane perpendicular to the axis of the hole.

$v \quad$ is Poisson's ratio.

$\alpha_{r} \quad$ is Biot's coefficient in the direction radial and perpendicular to the hole.

Equation (8) may be re-arranged to provide a solution for stresses based on the pilot hole deformation in overcoring.

If we examine Equation (8) we may note that it lacks any term related to pore pressure and Biot's coefficient in the axis of the hole. This actually disappears in the derivation.

\section{Which stress measurement method to use?}

Hydrofracture is simple in principle but suffers from complications in operation and interpretation. Its operation is complicated by the need to seal the borehole at each end of the hydrofracture zone. If packers are to be used for this purpose they need to be at a higher pressure than the fracture fluid, otherwise it will leak past them. If they are at a higher pressure then the likelihood is that they will initiate the fracture.

The petroleum industry tends to conduct such testing without the use of packers being in contact with the borehole wall. Testing is rather conducted through a casing that is cemented in place and perforated. This means that the test cannot be used for the determination of the major stress.

A great deal of work has however been expended by the petroleum industry in the analysis of mini-fracture closure pressure so as to determine the minor stress (Martin et al. 2012). The driver for this has been for the design of production hydrofractures for petroleum extraction. Theoretically this closure pressure is free from any of the complications related to Young's modulus, Poisson's ratio or Biot's coefficient.

The determination of the major principal stress from hydrofracture does however have a number of complications. In addition to the packer issues mentioned above it relies on linear elastic behaviour of the rock to give an analysable stress distribution around the hole. The fracture opening pressure is also strongly influenced by the Poisson's ratio and Biot's coefficient. Another factor that limits the determination of the major stress from the initial hydrofracture is the tensile stress of the rock. Subsequent fracture re-opening does not have to overcome the rock's tensile stress. However it relies on the assumption that the fracture closes perfectly and the rock around the hole behaves exactly as it did before the first hydrofracture. This is not generally the case as repeated fracture opening tend to show that the opening pressure is both related to the number of cycles of re-opening and to the flow rate of the pump. Basically the fracture does not close properly. Additionally the effects of repeatedly re-pressurising changes the effective stress within the zone around the hole. 
Overcoring has its limitations as well. Equation (8) is also based on elastic theory of the stress distribution around a hole. The solution of the far field stresses relies on a knowledge of Young's modulus and Poisson's ratio. Fortunately the mean stress (radial+tangential+axial) around a circular opening in a uniform stress field is theoretically constant and it would appear from triaxial testing for Young's modulus and Poisson's ratio that these are dependent on the mean stress that the rock is subject to. Hence a constant stress dependent value of the secant unloading Young's modulus and Poisson's ratio can be used in the analysis of the overcore. If the rock sample is too nonlinear in its stress strain behaviour then the accuracy of any overcore analysis using a solution based on a constant modulus will be limited.

Equation (8) may be broken into two parts. In addition to the effect of Young's modulus, the mean diameter change is affected by the mean horizontal stress perpendicular to the hole axis $\left(\sigma_{m}\right)$, the stress along the hole axis $\left(\sigma_{z}\right)$, normally taken as overburden stress, Poisson's ratio $(v)$, Biot's coefficient $\left(\alpha_{r}\right)$, and fluid pressure $(p)$. Thus the potential for errors in the mean stress grows with depth associated with the axial stress and fluid pressure. The deviatoric component of stress $\left(\sigma_{D}\right)$ may however be more accurately determined from the out of roundness changes to the hole diameter measured during overcoring and influenced only by the Young's modulus and one minus the square of Poisson's ratio, $\left(1-v^{2}\right)$. The latter remains reasonably close to unity even if the rock is totally plastic $(v=0.5)$.

Thus if we had had no financial constraints and really needed to be certain of the measurements of stress in a deep hole we might conduct overcoring while drilling a hole and check the minor stress by hydrofracture closure later. In measurements closer to surface overcoring appears superior, provided the rock is reasonably elastic, because the uncertainties related to fluid pressure and vertical stress become insignificant.

If the normal stress is to be measured across rock joints then hydrofracture is the only means to do this effectively.

\section{$7 \quad$ Effective stress in failure}

Most rock is jointed and failure will occur on joints. These joints are caused by failure of the rock mass at some point in the past. They may be open or have been subsequently deformed together or been infilled with a mineral.

Joints are essentially generally curved surfaces suited in concept to analysis by Equation (1). The question is what is $\alpha$ ? In a partially open joint it might be thought of as the fraction of joint area on which fluid pressure may act, or that is not in sealing contact. If however the joint is filled with calcite then how does this behave? Calcite deposited in a joint can be expected to have the same compressibility as the mineral itself and therefore the Biot's coefficient of this material can, according to Equation (4), be expected to be zero. This would suggest that changing fluid pressure has no influence on the effective stress within a joint filled with a crystalline mineral deposit. If however the joint fill material is clay from weathering then Biot's coefficient might be expected to be unity and therefore the fluid pressure will act fully.

Some weak minerals may however exist in the unaltered state. For example a zone of talc or chlorite may never have been sheared and be a consistent crystalline structure. In this case we may hypothesise it as having a Biot's coefficient of zero. No measurement of Biot's coefficient has apparently ever been made on an undisturbed sample of these materials. If however rock movement has taken place then the value of Biot's coefficient may have dramatically changed.

The same question may be asked about fractured material which has deformed so that the sides of the fracture are in intimate contact. How does fluid pressure act within these fractures to change the effective stress? Presumably it will behave as in the body of the adjacent rock.

The adherents of rock mass quality approaches (Barton et al. 1974) to the design of rock structures take into account the number, infill, openness and water pressure in rock joints. Open joints are more of a concern for stability than closed ones. Open and wet joints are of still more concern, particularly if the joints start to open and link up. Such an empirical approach is consistent with a changing area ratio, similar in application to Biot's coefficient, over which fluid pressure may act. 
Failure in a rock mass is essentially a process of fracture formation and opening until an unacceptable level of deformation occurs. This may be excessive movement in a tunnel, a collapse or a rockburst. The fractures may be in virgin rock but are more usually pre-existing joints in various states of openness. This movement is dependent on effective stress. The actual failure, in terms of breakage, is a coalescing of fractures on some surface or surfaces in the rock mass. This fracture interconnection is accompanied by a change in the effective area ratio of the failure surface subject to fluid pressure.

Wood and Gray (2015) came to the conclusion that outbursting in coal may be considered to be a failure associated with the development of pre-existing fracture surfaces that were in tight contact. This was followed by fragmentation and energy release. These pre-existing fractures form the basis of breakage within the outburst. The outburst is gas driven and the main energy release will usually come from gas expansion from the gas. The question is how the fluid acts within these pre-existing planes prior to and during the outburst? This may be expected to be a function of Biot's coefficient within the coal. To date Biot's coefficient is not been considered in the outburst literature.

It would appear that the pre-existing fractures are planes of weakness on which failure starts. As failure progresses these fractures open and gas desorbs from the coal into these. Because the gas is desorbing and highly compressible it maintains its pressure during the early stages of failure until the fractures open to the face thus permitting the pressure to leak off. In this case the mechanism of the outburst is one of failure followed by propulsion due to strain energy release, propulsion from expanding gas between opening planes and finally due to particle entrainment in the gas stream.

\section{$7 \quad$ Concluding remarks}

Effective stress matters. It is the key to understanding deformation and failure of a rock mass. Effective stress is the total stress minus the product of fluid pressure and Biot's coefficient.

This paper addresses the dual nature of Biot's coefficient in rock mechanics. The basic equation of effective stress includes Biot's coefficient in the form of an area ratio over which fluid pressure may act. As such it has immediate relevance to a failure surface. This may be a surface of negligible thickness in which case the concept of poroelasticity does not apply. This is an unconventional use of the parameter and possibly the term Biot's coefficient should not be used in its description.

The more conventional view of Biot's coefficient is as a poroelastic parameter. The measurement of it must somehow involve deformation. This has hitherto been by volumetric change within a stressed sample that produces a scalar value. Biot's coefficient can be mathematically described as a tensor and its directional behaviour measured. A method to do this is presented in this paper. Biot's coefficient is also stress dependent becoming greater with reducing stress.

In addition to the increasing Biot's coefficient with reducing stress in the solid, once fractures open up their full area can be expected to be under the influence of any fluid pressure. Thus the effective stress changes in a rock mass may be thought to be a function of both fluid pressure and the changing Biot's coefficient, whether it is considered as an area ratio, or as poroelastic response. In either case the coefficient is directionally dependent, either on the fracture or fabric of the rock and its intersection with the plane being considered.

Stress measurement is also revisited. Obviously effective stress is a direct function of Biot's coefficient. However its value also affects the measurement of stress by hydrofracture and overcoring. A possible combination of the two stress measurement systems may offer the most certainty in determining stress at depth.

It is necessary to consider Biot's coefficient in the analysis of a rock mass. This means that it must be adequately estimated or better, measured, something that is sadly lacking in current practice. The process described for the determination of Biot's coefficient using strain gauged core which is loaded externally and internally by fluid pressure has significant advantages over the purely volumetric approaches generally used. This applies especially to the determination of the directional values of Biot's coefficient in anisotropic rocks. 
As effective stress controls the deformation of a rock mass all models dealing with rock should be written in terms of effective stress. This applies just as much to a slopes as to deep mine openings or to the use of hydrofracturing for the production of petroleum fluids.

\section{Acknowledgement}

The author wishes to thank Bill Daniel and Ben Seeto for their suggestions regarding the mathematics of this paper. Xiaoli Zhao and Lucy Liu are thanked for testing and analysing the results on Sydney sandstone to produce a value of Biot's coefficient.

\section{References}

Barton, NR, Lien, R \& Lunde, J 1974, 'Engineering classification of rock masses for the design of tunnel support', Rock Mechanics and Rock Engineering, vol. 6, no. 4, pp. 189-236.

Betti, E 1872, Il nuovo cimento, ser. 2, vol. 7-8.

Biot, MA \& Wills, DG 1957, 'The elastic coefficients of the theory of consolidation', Journal of Applied Mechanics, vol. 24, pp. 594-601.

Detournay, E \& Cheng, AH-D 1993, 'Fundamentals of poroelasticity', in C Fairhurst (ed.), Comprehensive Rock Engineering: Principles, Practice and Projects, Volume 2: Analysis and Design Methods, Pergamon Press, pp. 113-171.

Franquet, JA \& Abass, HH 1999, Rock Mechanics for Industry, in B Amadei, RL Kranz, GA Scott \& PH Smeallie (eds), Balkema, Rotterdam, pp. 349-355.

Gray, I 2000, 'The measurement and interpretation of stress', in JW Beeston (ed.), Bowen Basin Symposium 2000: The New Millennium - Geology Proceedings, 22-24 October 2000, Rockhampton, Geological Society of Australia Coal Geology Group.

Gray, I, Wood, JH \& Shelukhina, I 2013, 'Real stress distributions in sedimentary strata', Proceedings of the 6th International Symposium on In-situ Rock Stress, 20-22 August 2013, Sendai, International Society for Rock Mechanics, Lisboa.

Haimson, B \& Fairhurst, C 1969, 'In-situ stress determination at great depth by means of hydraulic fracturing', in W Somerton (ed.), Proceedings of The 11th U.S. Symposium on Rock Mechanics, American Institute of Mining Engineers, New York, pp. 559-584. Isaac, MD \& Ori, I 2006, Engineering Mechanics of Composite Materials, 2nd edn, Oxford University Press, Oxford.

Martin, AR, Cramer, DD, Nunez, O \& Roberts, NR 2012, 'A method to perform multiple diagnostic fracture injection tests simultaneously in a single wellbore', 2012 SPE Hydraulic Fracturing Technology Conference, 6-8 February 2012, Woodlands, Society of Petroleum Engineers, pp. 191-200.

Tezaghi, K \& Peck, RB 1948, Soil Mechanics in Engineering Practice, John Wiley \& Sons, New York.

Wood, JH \& Gray, I 2015, Outburst risk determination and associated factors, report on Project C23014 for the Australian Coal Industry Research Program, Brisbane. 
